\title{
Research on Establishment Method of Index System for Weapons and Equipment Operational Test
}

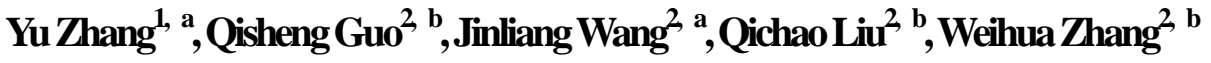 \\ ${ }^{1}$ Eight one road Houairou District, Beijing, China \\ ${ }^{2}$ Dujiakan 21 Fengtai District, Beijing, China

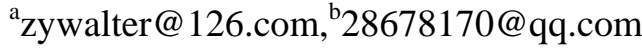

Keywords: Equipment operational test; Operational adaptability; Operational effectiveness

\begin{abstract}
The establishment of an equipment operational test index system is the basis for operational test design, implementation and comprehensive evaluation. To solve the problems such as operation procedure is unclear and not easy to operate, and promote the effective conduct of the equipment operational test practices, the paper proposes many operational test question from equipment users and mission tasking order, decomposes operational test question on the base of many methods, establishes and combines index system, and establishes complete steps of operational test index system.
\end{abstract}

\section{Introduction}

At present, our military is vigorously carrying out theoretical research on weapon and equipment operational test, and evaluating the index system as the base point of operational test design, implementation and comprehensive evaluation [1]. Whether the construction method is scientific, whether the content setting is comprehensive and reasonable is directly related to the success or failure of the operational test. Therefore, the construction of the operational test evaluation index system has become a research hotspot in the field of military equipment. For example, Yixin Xue[2] proposed starting from the key operational issues, and establishing operational effectiveness and operational suitability index through the analysis of its functions and capabilities from top to bottom; Liang Wang[3] studied the basic concepts and connotations of operational test indicators, and laid the foundation for the construction of operational test index system; Xiaofeng Zhang[4] changed the mesh index system based on the idea of "copy-distribution" to the tree indicator system. The above research results have studied the operational test evaluation index system from different angles, but have not formed a general, complete and operable construction steps. Based on the research on the type of operational evaluation index system and the construction principle, this paper gives the steps to construct the evaluation index system, focusing on the comprehensive evaluation index system.

\section{Operational test Evaluation Index System Type}

The evaluation index system differs according to the purpose of the test, the type of test equipment, and the construction method of the indicator system. It is usually classified according to the structure of the indicator system and the content of the test.

\subsection{According to the Structure.}

The corresponding evaluation index system is established by analyzing the functional level, structural level and logical level of the equipment system. Such evaluation indicator system has the characteristics of clear structure and simple and easy to use, and generally includes three levels: target layer, criterion layer and scheme layer. There is usually only one target layer, which is generally vague, general and abstract. It is not convenient to quantify and measure. For this reason, the target layer is decomposed into various levels of criteria and sub-standards until it is quite specific and intuitive, and can be applied directly or indirectly. It isn't stopped until the indicator of 
the program is characterized. In the hierarchical structure, the lower-level criteria are more specific than the upper-level criteria and are easy to compare, judge, and measure. The indicators at each level are relatively independent. The hierarchical structure of the evaluation index system is shown in Fig. 1.

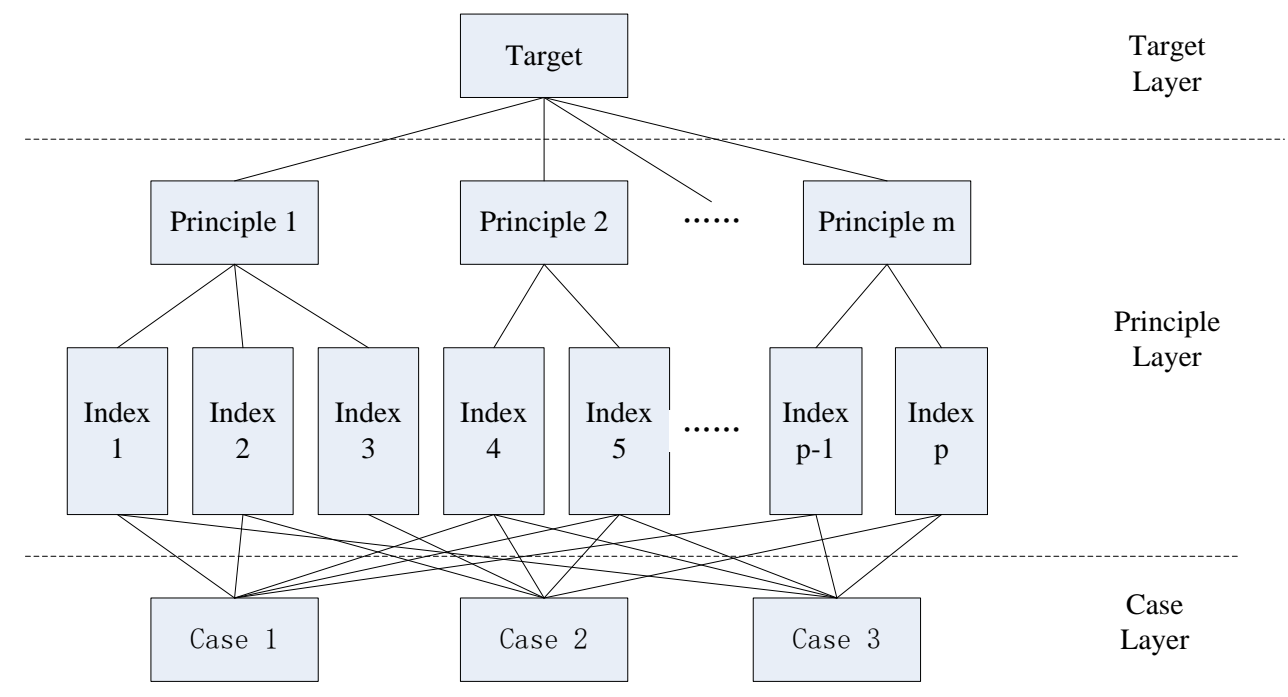

Fig. 1 Evaluation index system hierarchy structure

For some high-tech equipment, due to the complexity of the internal structure, it is often difficult to separate the evaluation indicators or the system evaluation model itself has not yet been determined, and the network-like evaluation index system should be used or partially used. A certain level of the system can be in a dominant position and can be directly or indirectly accepted by other levels, both in a hierarchical structure and in a dominant structure. The network structure consists of two major parts: the control layer and the network layer. The control layer includes the problem target and the decision criterion. It is the top layer in the network hierarchy. The rules in the layer are relatively independent. The next criterion is only subject to the previous criterion. The network layer is composed of all the elements controlled by the controlled layer. Independent of each other and interacting, an element is likely to affect any set of elements in the entire network system, and vice versa. The network structure of the evaluation indicator system is shown in Fig. $2[5]$.

\subsection{The Test Content.}

Operational effectiveness refers to the extent to which operational forces play an effective role in the operational process. Based on the reductionism and holistic theory, the distinction is made from two perspectives: operational capability and operational effectiveness.

Based on the idea of reductionism, the tree decomposition technique is used to decompose layer by layer. The upper layer and the lower layer are inclusive, and the same layer indicators are relatively independent, as shown in Fig. 3.

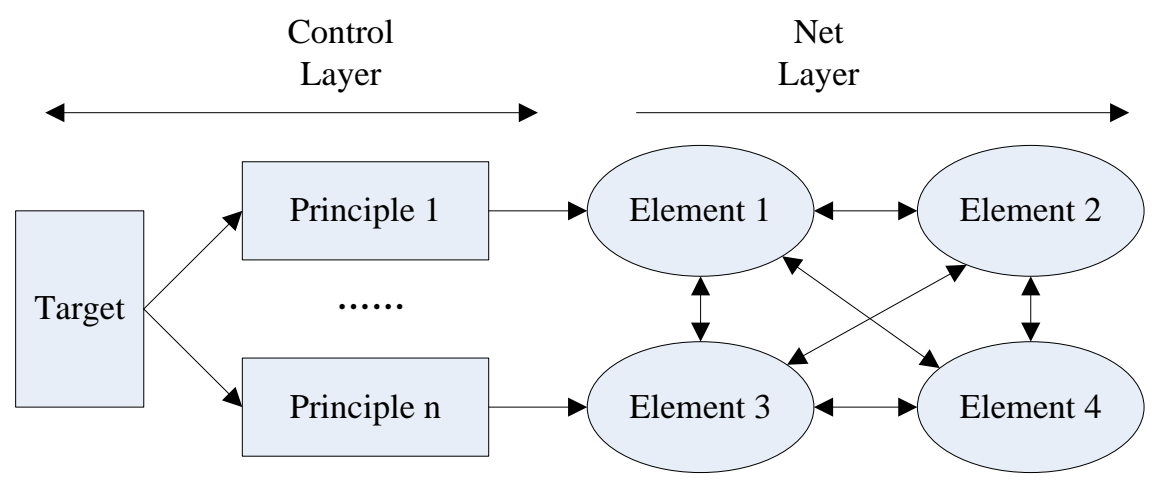

Fig. 2 Evaluation indicator system network structures 


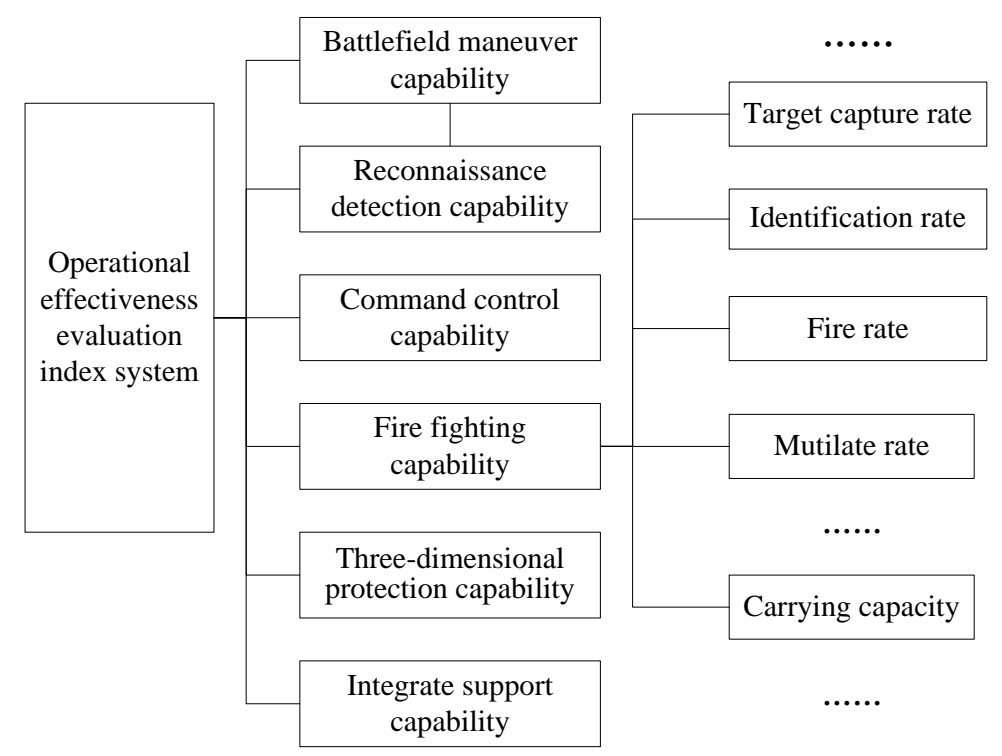

Fig. 3 A certain type of armored operational effectiveness evaluation index system based on operational capability

Based on the holistic theory, the assessment [6] is carried out in terms of operational duration, damage to our equipment, damage to enemy equipment, consumption of our ammunition, and consumption of enemy ammunition, as shown in Fig. 4.

Operational suitability refers to the extent to which weapons and equipment can remain available during operational use. The focus is on the two aspects of affecting the operational effectiveness of the equipment and the satisfaction of the equipment. The classification of the evaluation indicators is as follows:

Based on the "three-capability" operational appraisal evaluation index system. That is to say, it is assessed from three aspects: the suitability of the operational environment, the applicability of operational use, and the applicability of operational support [7].

Example 1: The operational applicability indicators of a vehicle-mounted missile weapon system are as follows:

Applicability: The ability to prevent fatal failures during the operational process and ensure the normal completion of the mission; good protection measures to better protect personnel safety and reduce their own loss; better operational convenience and lower operation The ability to make mistakes and improve operational efficiency.

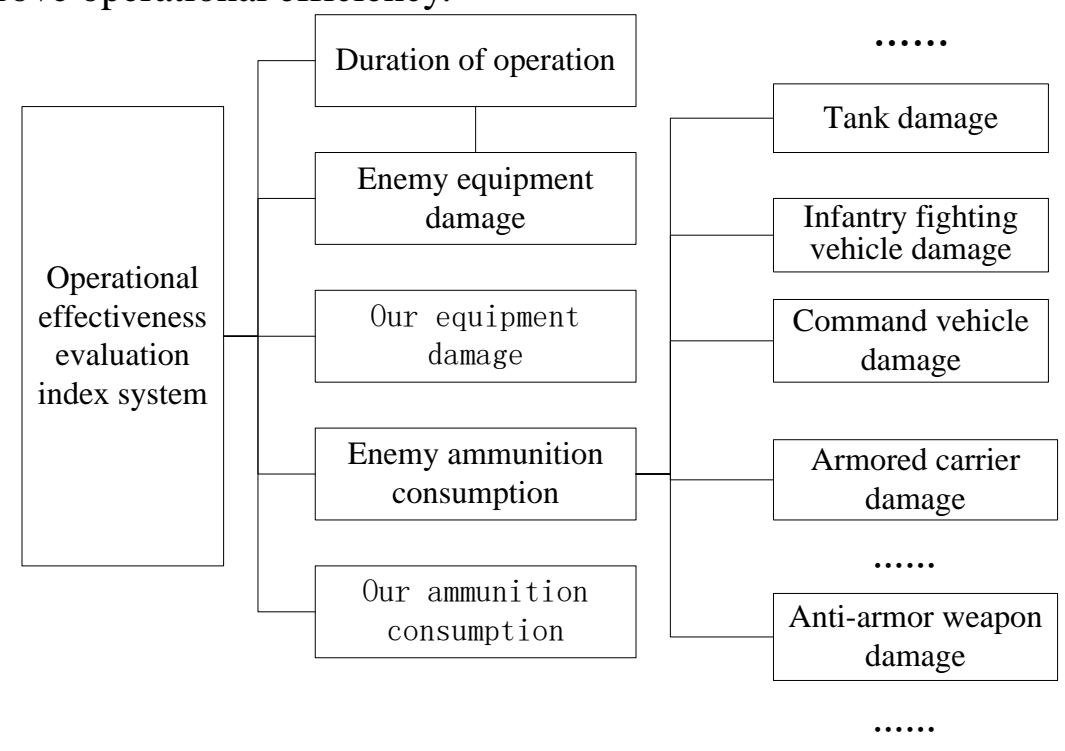

Fig. 4 Operational effectiveness evaluation index system based on operational effectiveness 
Environmental adaptability: adapt to the typical operational direction of China, such as the plateau, alpine, desert, jungle, coastal and other typical harsh environments.

Assurance applicability: The availability of operational readiness can be implemented; the guaranteed resources can meet the requirements of normal training, operational readiness and operations; it can meet the requirements of quickly detecting faults and performing repairs and repairs.

Based on the "five-capability" operational applicability index system. That is, the assessment is based on five aspects: suitability of the operational environment, applicability of operational missions, suitability of operational support, suitability of operational preparation, and applicability of ergonomics.

Example 2: The operational suitability indicators for a certain type of electronic equipment are as follows:

Suitability of the operational environment: adaptability to the natural environment of the battlefield, the combined firepower environment, and the electromagnetic environment of the battlefield.

Operational Task Adaptability: operational integrity and continuous operational capability during mission execution.

Applicability of operational support: including the guarantee of operational maintenance and operational support resources.

The suitability of operational is compiled: Including the suitability of personnel and the suitability of equipment.

Ergonomic applicability: Including software and hardware man-machine interface operation convenience, operational use security, man-machine combination, man-machine security.

Based on the "ten-capability" of operational applicability index system. That is, the applicability of the equipment is evaluated in terms of availability, reliability, security, maintainability, testability, transportation, compatibility, survivability, safety and human-machine adaptability.

Example 3: The main operational applicability indicators of a tank rescue vehicle are as follows:

Reliability. In the process of carrying out the accompanying support task, there is no failure that affects the completion of the support task.

Maintainability. Its own fault detection and inspection adjustment is convenient and quick, the maintenance accessibility is good, and general faults can be eliminated by the grassroots repair organization.

Security. The equipment, tools and accessories are complete and applicable; the technical information of the installation is complete and accurate, and can guide the use and maintenance of the tested products; the use and maintenance of the equipment meets the operational requirements of the troops.

Safety. During the operation of the rescue, traction and delivery, there is no accident caused by the equipment.

Human-machine adaptability. The operation is convenient, the space layout is reasonable, the human-machine interface is friendly, and the working environment meets the operational requirements.

\section{Operational Test Evaluation Index System Construction Principles}

In addition to meeting the general principles of systematicness, conciseness, objectivity, and timeliness, the evaluation index system must also consider the degree of demand for operational missions.

\subsection{Integration of Comprehensiveness and Emphasis.}

In order to thoroughly understand the test equipment base, the evaluation index system is built to be comprehensive. However, it is necessary to avoid duplication and unnecessary waste in all aspects. This requires that the evaluation indicators corresponding to different experimental stages should be clear, the connotation should be clear, and the assessment key should be prominent. 
Assume that the tank battalion's offensive operational process is divided into four stages: assembly, advancement, deployment, and operational. The assembly phase focuses on the assessment of long-distance transport capability and campaign maneuverability; the advancement and deployment process focuses on tactical maneuverability and command and control capabilities; Focus on the firepower strike capability and comprehensive protection capability during the operational phase.

\subsection{The Fusion of Test Tasks and Mission Tasks.}

Weapons and equipment operational test is to comprehensively test the ability of weapons and equipment to complete operational missions in a near-real operational environment. Different types of equipment usually include different types of operational troops, and carry out various operational missions, corresponding to different operational mission profiles. As the basis of conducting operational tests, the index system should be based on the actual operational background, embody the test tasks on the basis of completing the operational missions, and highlight the actual operational test.

\subsection{The Fusion of Equipment Development and Capacity Needs.}

Equipment development stems from capacity needs, and when equipment cannot meet operational requirements, the demand for equipment capabilities arises. Therefore, based on the "capability demand analysis" idea to establish a operational test evaluation index system, not only to study the future operational style proposed capacity concept, but also to consider the specific problems found in the daily training and exercises of the equipment users, to some extent make the index system build more Forward-looking and targeted.

\section{Evaluation Index System Construction Steps}

In order to ensure that the evaluation index system is scientific and reasonable, the index system construction unit comprehensively considers the equipment use object and equipment mission and mission requirements according to the general requirements of equipment development, and proposes weapon equipment characteristics, multi-task requirements/specific operational mission requirements, and key operational issues. The evaluation indicators are initially determined based on mapping, indicator function deployment, and tree analysis. In view of the unclear level of indicators and the complex relationship between indicators and indicators, based on Interpretative Structural Modeling (ISM), the network index system is transformed into a tree index with good hierarchical structure and relatively independent index relationship. The system, combined with the operational tasks, refines the evaluation index system. The overall construction idea is shown in Figure 5.

\subsection{Analysis of Operational Test Questions.}

Analysis of the operational test problem is the basis and premise of the evaluation of the index system. By participating in the preparation of equipment demonstration and development requirements, understand the operational mission and mission of the equipment, the main tactical technical indicators and the use requirements, and clarify the overall objectives of the operational test. Focus on two aspects of demand: First, the needs of equipment users, the new research equipment must be distributed to the operational troops for daily training, exercises and operations, which is particularly important for the equipment's opinions and functional requirements, and directly related to the operational effectiveness of the troops. Second, equipment missions, it is especially important to have specific functional requirements in different test equipment, and it is directly related to the improvement of operational effectiveness of the troops. Second, it is equipped with mission tasks. Different test equipments have their specific operational missions. From the operational missions, they think about how to build an indicator system, which can be combined with the overall objectives of the operational test. Consistently, to better reflect the idea of trial integration. 


\subsection{Proposed Operational Test.}

The operational test problem is the specific of the needs of equipment users and equipment mission tasks. The relatively scattered or fuzzy requirements are transformed into relatively clear equipment characteristics, functional requirements, mission indicator requirements or key operational issues, laying the foundation for the subsequent index system construction.

The equipment characteristics are usually proposed by the equipment user, and the content of the demand is relatively scattered, usually including key performance parameters, key system characteristics, other system characteristics, etc. According to the correspondence between the combat mission and the equipment function, various functional requirements, such as firepower function requirements, can be proposed. command and control function requirements, battlefield maneuverability requirements, etc; according to specific combat mission requirements (such as an air defense missile position to identify and destroy the probability of attacking enemy aircraft to 98\%), determine other levels of indicators requirements; according to operational test objectives, Propose key operational issues, such as whether a certain type of equipment has superior maneuverability. Does a certain type of equipment have an absolute firepower advantage in the fire assault phase?

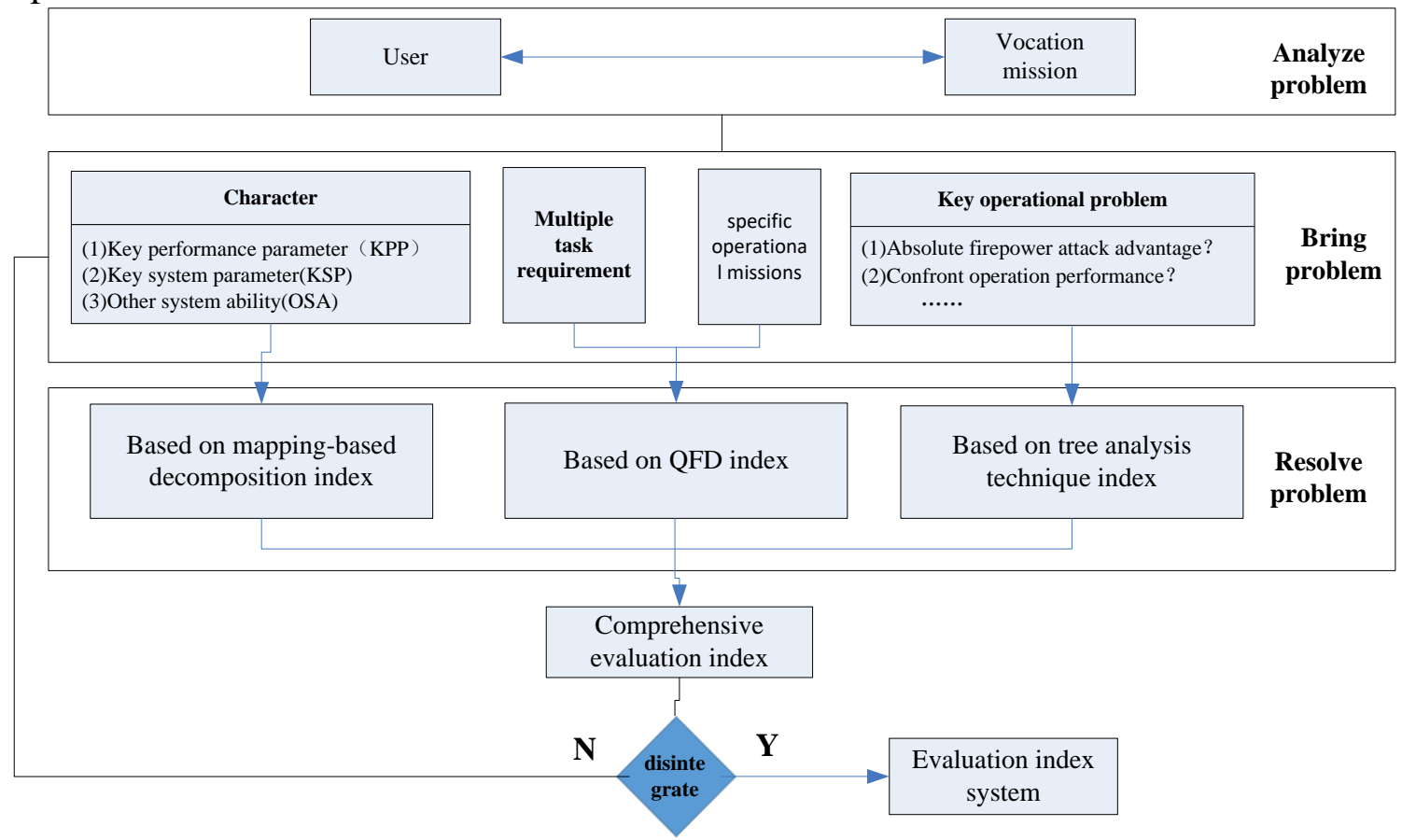

Fig. 5 Construction steps of the weapon equipment operational test evaluation index system

\subsection{Analysis of Operational Test Problems.}

The essence of analyzing operational problems is to use a variety of decomposition methods to initially transform the operational test problems into an evaluation indicator system.

A mapping-based decomposition method. It was mainly used to transform weapon equipment characteristics into an evaluation indicator system. The specific process includes 2 steps: the first step gives the equipment characteristic index, the combat effectiveness and the operational application, lists the rows and columns of the mapping table; the second step corresponds to the row and column, and plays in the cross box. $\sqrt{ } "$, so that we can classify the equipment characteristics indicators, it is shown in Table 1.

A decomposition method based on QFD. Mainly used for multi-task and specific combat mission requirements to be converted into an evaluation indicator system. QFD is a multi-level deductive analysis method 9 based on maximizing customer satisfaction during product development. It can transform multi-task and specific combat mission requirements into quality requirements. The specific process includes three steps: the first step is to decompose the task profile and complete the 
combat mission. $\rightarrow$ The mapping of operational activities can also give the order of importance of combat missions and operational activities; the second step is to fulfill the operational requirements of different operational activities. $\rightarrow$ The mapping of combat capability; the third step is based on the basic functions of the equipment, and is decomposed to meet the capability requirements to complete the combat capability. $\rightarrow$ While evaluating the mapping of indicators, an evaluation indicator system[8] was also constructed.

Table 1 Weapon equipment characteristics mapping table

\begin{tabular}{|c|c|c|c|}
\hline Type & Index & Operational effectiveness & Operational suitability \\
\hline \multirow[t]{4}{*}{ KPP } & Indicator 1 & $\sqrt{ }$ & \\
\hline & $\ldots$ & & $\sqrt{ }$ \\
\hline & Indicator m & $\sqrt{ }$ & \\
\hline & Indicator 1 & $\sqrt{ }$ & \\
\hline \multirow[t]{3}{*}{ KSA } & $\ldots$ & & $\sqrt{ }$ \\
\hline & Indicator $\mathrm{n}$ & $\sqrt{ }$ & \\
\hline & Indicator 1 & & $\sqrt{ }$ \\
\hline \multirow[t]{2}{*}{ OSA } & $\cdots$ & $\sqrt{ }$ & \\
\hline & Indicator $\mathrm{n}$ & $\sqrt{ }$ & \\
\hline Remarks & $\begin{array}{l}\text { KPP represe } \\
\text { system charac }\end{array}$ & $\begin{array}{l}\text { ey performance parameter } \\
\text { tics; OSA represents other }\end{array}$ & $\begin{array}{l}\text { KSA represents key } \\
\text { ystem characteristics. }\end{array}$ \\
\hline
\end{tabular}

Decomposition method based on tree analysis technique. It is mainly used to decompose the evaluation index system for key operational problems. The tree analysis technique originated from the reduction theory. It is a methodology that advocates the study of the whole decomposition. It can gradually transition the abstraction level to the specific details, thus expressing the various attributes and states of complex objects. The essence of the decomposition method based on tree analysis technology is to gradually decompose key problems into a clear tree structure; from high-level to low-level, which are key operational issues, performance/applicability indicators, performance indicators and required data elements [9].

\subsection{Comprehensive Evaluation Index System.}

When the level of evaluation indicators after analysis is unclear and the relationship is complex, it is necessary to adopt a certain method for relationship analysis and processing. Based on Interpretative Structural Modeling (ISM), this paper transforms the network evaluation index system into an intuitive tree structure evaluation index system with good hierarchy.

Establishing a triangular matrix. The triangular matrix is used to describe the relationship between the evaluation elements. The purpose is to compare the evaluation factors that are not hierarchical and not conducive to the analysis of the operational test questions, and analyze the relationship between the evaluation elements. The relationship between the evaluation elements mainly includes the following four types:

$\mathrm{S}_{\mathrm{i}} \times \mathrm{S}_{\mathrm{j}}$, There is relationship between $S i$ and $S j$;

$\mathrm{S}_{\mathrm{i}} \varnothing \mathrm{S}_{\mathrm{j}}$, There is no relationship between $S i$ and $S j$;

$\mathrm{S}_{\mathrm{i}} \cup \mathrm{S}_{\mathrm{j}}, S i$ is related with $S j$, but $S j$ is irrelevant with $S i$;

$S_{i} \cap S_{j}, S_{i}$ is irrelevant with $S_{j}$, but $S_{j}$ is related with $S_{i}$.

Taking a certain type of armored equipment operational test as an example, by analyzing the operational test problems, the following indicators are proposed for the battlefield maneuverability: combat maneuverability, maneuver distance, average maneuver speed, tactical maneuverability, traffic capacity and flexibility. These indicators have the characteristics of unclear subordinate hierarchy and unclear relationship between indicators. A comparative triangular matrix is established, and the relationship is established from bottom to top. The following evaluation elements are $S i$, the above evaluation elements are $S j$. 
Establishing a reachable matrix. The purpose is to judge the complexity of the relationship between the indicators. The reachable matrix is usually obtained by the operation of the adjacency matrix, that is, the adjacency matrix plus the unit matrix can be obtained after at most (n-1) power operation. If the characteristics of the reachable matrix are obvious, they can also be derived directly. Horizontal evaluation factorSi Vertical evaluation factorSj in comparison, when the comparison relationship belongs to the first and third species, the corresponding value is 1 ; when the comparison relationship belongs to the 2nd and 4th, the corresponding value is $0[10]$ and the reachable matrix $\mathrm{M}$ is as follows:

$$
M=\left[\begin{array}{cccccccc}
\multicolumn{1}{c}{S_{0} S_{1} S_{2} S_{3} S_{4} S_{5} S_{6}} \\
S_{0} & 1 & 0 & 0 & 0 & 0 & 0 & 0 \\
S_{1} & 1 & 1 & 0 & 0 & 0 & 0 & 0 \\
S_{2} & 1 & 0 & 1 & 0 & 0 & 1 & 0 \\
S_{3} & 1 & 0 & 1 & 0 & 0 & 0 & 1 \\
S_{4} & 1 & 1 & 1 & 0 & 0 & 0 & 0 \\
S_{5} & 1 & 1 & 1 & 1 & 0 & 0 & 0 \\
S_{6} & 1 & 1 & 1 & 0 & 1 & 0 & 0
\end{array}\right](1)
$$

Generating a hierarchical directed graph. The reachable matrix $\mathrm{M}$ is not regular, and it is still impossible to explicitly evaluate the feature hierarchy. This requires further processing of the reachable matrix to generate a matrix in which the elements in the upper right corner are all zero. According to the order of 1 in each row of elements, the rows and columns are rearranged to form a matrix in which the elements in the upper right corner are all $0 . M^{\prime}$, as follows:

$$
M^{\prime}=\left[\begin{array}{cccccccc}
\multicolumn{1}{c}{S_{0} S_{1} S_{4} S_{2} S_{3} S_{5} S_{6}} \\
S_{0} & 1 & 0 & 0 & 0 & 0 & 0 & 0 \\
S_{1} & 1 & 1 & 0 & 0 & 0 & 0 & 0 \\
S_{4} & 1 & 0 & 1 & 0 & 0 & 0 & 0 \\
S_{2} & 1 & 0 & 1 & 0 & 0 & 1 & 0 \\
S_{3} & 1 & 0 & 1 & 0 & 0 & 0 & 1 \\
S_{5} & 1 & 1 & 1 & 1 & 0 & 0 & 0 \\
S_{6} & 1 & 1 & 1 & 0 & 1 & 0 & 0
\end{array}\right](2
$$

It can be seen that the upper right part is zero by Matrix $M^{\prime}$, and the corresponding evaluation element is the maneuvering distance $S_{2}$, average maneuver speed $S_{3}$, capacity $S_{5}$, and flexible mobility $S_{6}$. It shows that the four evaluation indicators are at the bottom of the indicator system, the battlefield maneuverability is at the top level, and other evaluation factors are temporarily placed in the middle layer.

The purpose of model map transformation is to transform the network evaluation index system into a tree structure by means of copy-allocation. The core idea is to eliminate the problem that the next level of elements has a relationship with multiple upper level elements, thus simplifying the indicator system structure. All subordinate elements that are active at the same time are copied, and the result of the copy is placed under different upper level elements until the bottom element acts on only one upper level.

After the model indicator transformation, the same indicator name appears, but the specific meaning of each indicator is not the same. It is necessary to continue to refine the indicators according to the test purpose and the operational profile until the meaning is clear.

For the sub-indicators under the operational maneuverability, the capacity refers to the degree to which the new equipment is adapted to the methods of rail transport, air transport, sea transport, land transport, etc., and is embodied by dimensional applicability, weight applicability, and fixed applicability; Maneuverability refers to the convenience of loading equipment, which is reflected by the loading and unloading time of the end station and the emergency unloading time.

For the sub-indicators under the tactical maneuverability, the traffic capacity refers to the ability to climb the slope with a large slope and the turning rate of the large angle through the specific 
terrain environment capability in the combat area; the maneuver distance refers to the test equipment. The specific combat terrain, maneuverability under full oil conditions, is reflected by the continuous maneuver distance; the average maneuver speed refers to the maneuverability under different weather conditions in the combat area, the average maneuver speed of the march in the specific combat area, the average maneuver speed at night, and the rainy day. The average maneuvering speed of the march is reflected in the day; the flexible maneuverability refers to the ability of the combat unit as the main unit of the test equipment to evacuate and conceal, the formation and transformation of the combat formation, the evacuation time, the reconnaissance effect under different reconnaissance means, and the formation of the combat formation.

Take the mountain jungle tanks as an example of offensive operations. After refinement and improvement, the battlefield maneuverability index system is shown in Fig. 6.

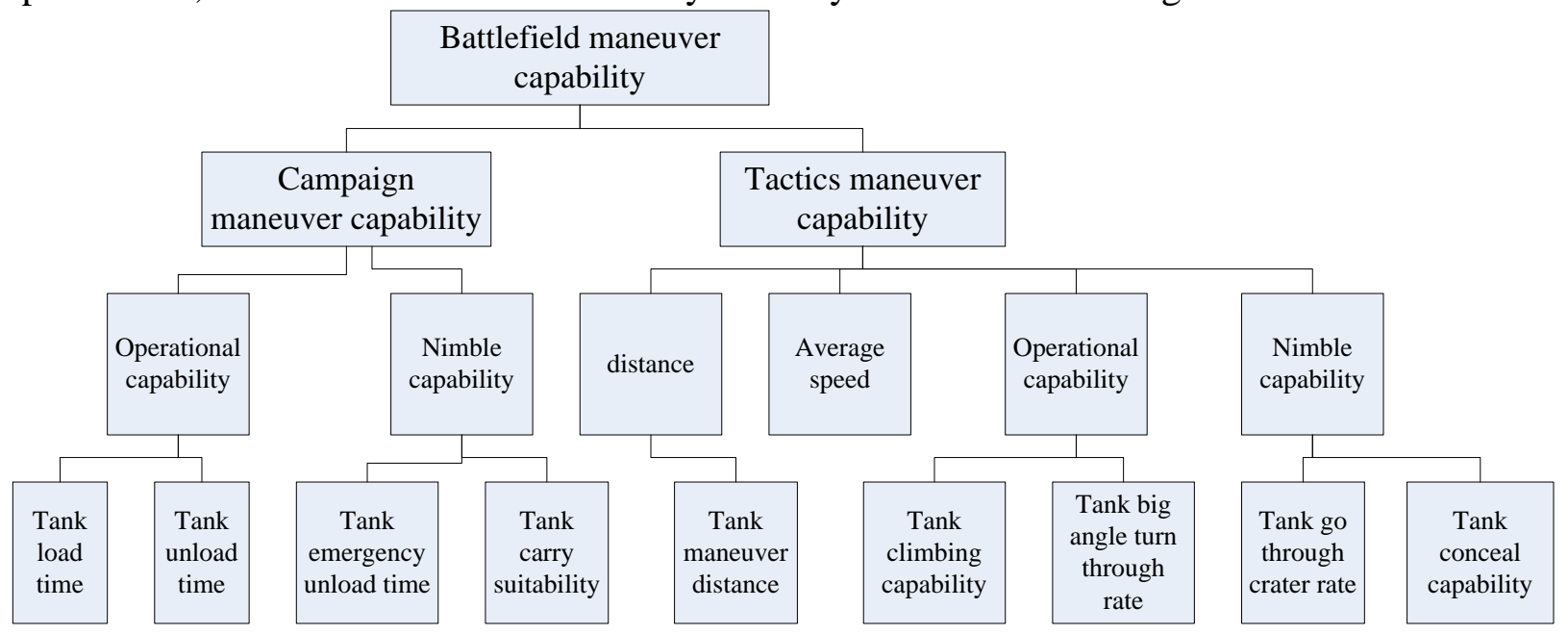

Fig. 6 Evaluation index system for the maneuverability of the battlefield in the jungle

\section{Conclusion}

The construction of the evaluation indicator system is a complex system engineering, and many factors need to be considered, involving multiple subject areas. Whether the index system is constructed scientifically or not has a great impact on the results of the operational test. This paper gives an idea and method for the construction of the evaluation index system. Its scientific rationality needs to be tested in practice. The next step is to focus on the evaluation and evaluation of the evaluation index system.

\section{References}

[1] H.F.Ke, Y.L.Zhu, and J.Q.Zhang. Four-domain perspective of electronic equipment operational effectiveness evaluation index system framework [J]. Journal of the Academy of Equipment, 2016,27(3):113-119. (In Chinese)

[2] Y.X.Xue, and W.Zhou. Establishment Method of Weapon Equipment Operational Test Identification Index System [J]. Journal of the Academy of Equipment, 2016, 27(4): 102-107. (In Chinese)

[3] L.Wang. Research on the Basic Concept and Connotation of Weapons and Equipment Combat Test Indicators [J]. Journal of the Academy of Equipment, 2015,26(3):121-125. (In Chinese)

[4] X.F. Zhang, H.P.Yan, and Z.P.Ren. A Method for Establishing Tree Evaluation Index System [J].Fire Control \& Command Control, 2007, 32(6): 77-79. (In Chinese)

[5] Y.L.Ma, Q.F.Shao, and M.Sun et al. Evaluation theory and method and its military citation [M]. Beijing: National Defence Industry Press, 2013: 13-15. (In Chinese)

[6] J.L.Wang, Q.S.Guo, and Y.S.Li et al. Overall thinking of the Army's equipment combat test[J]. 
Journal of Armored Force Engineering College, 2016, 30(3): 1-6. (In Chinese)

[8] B.Z.Zhang and Q.S.Guo. The theory and method of army weapons and equipment demand demonstration [M]. Beijing: National Defense Industry Press, 2012: 37-39. (In Chinese)

[9] Y.H.Cao, W.W.Zhou and H.M. Gao. Research on the Design of Weapon Equipment Combat Test Content [J].Journal of the Academy of Equipment, 2014, 25(4): 112-117. (In Chinese)

[10] Y.L.Wang System Engineering Theory, Method and Application [M]. Beijing: Higher Education Press, 1998: 35-40. (In Chinese) 